\title{
Emergence of Motor Synergy in Vertical Reaching Task via Tacit Learning
}

\author{
Mitsuhiro Hayashibe $^{1}$ and Shingo Shimoda ${ }^{2}$
}

\begin{abstract}
The dynamics of multijoint limbs often causes complex dynamic interaction torques which are the inertial effect of other joints motion. It is known that Cerebellum takes important role in a motor learning by developing the internal model. In this paper, we propose a novel computational control paradigm in vertical reaching task which involves the management of interaction torques and gravitational effect. The obtained results demonstrate that the proposed method is valid for acquiring motor synergy in the system with actuation redundancy and resulted in the energy efficient solutions. It is highlighted that the tacit learning in vertical reaching task can bring computational adaptability and optimality with modelfree and cost-function-free approach differently from previous studies.
\end{abstract}

\section{INTRODUCTION}

Our skeletal system has complex series of linkages that produce coupled dynamics. For instance, when we quickly move our forearm by flexing the elbow joint, the flexion torques on the elbow joint accelerate our forearm. However, due to the forearm's inertia, this acceleration produces torques also on the shoulder. These interaction torques have the undesired effect of accelerating the upper arm segment. The dynamics of multijoint limbs often causes such complex torques. However, the able-bodied subject can normally handle such interaction torques with motor learnig and its prediction without any problem [9][2]. Vertical reaching task was studied in both patients with cerebellar lesions and control subjects in [1]. They concluded that cerebellar patients had specific deficits in their predictive compensation for the interaction torques. In control subjects, the elbow and shoulder joints rotated in synergetic way to compensate the interaction torques [4].

It is known that Cerebellum takes important role in such motor learning by developing the internal model while comparing the actual outcome to the predicted outcome [5][14]. Feedback-error-learning (FEL) is well studied to bring computational adaptation paradigms, including prism adaptation, saccade adaptation and reaching [7][6]. There is extensive evidence that the learning system using feedback error relies on the cerebellum.

FEL can reduce the average error to zero, but once this is achieved, it doesn't provide a mechanism to systematically improve performance further such as minimizing total energy or torque changes [13] especially in the case of redundant

This work was supported by Toyota Motor Co.

${ }^{1} \mathrm{M}$. Hayashibe is with the DEMAR Project, INRIA Sophia-Antipolis and LIRMM, CNRS University of Montpellier, Montpellier, France mitsuhiro.hayashibe at inria.fr

${ }^{2} \mathrm{~S}$. Shimoda is with BSI-Toyota Collaboration Center, RIKEN, Nagoya, Japan shimoda at brain.riken.jp system. Thus, additional mechanism has been introduced such as optimization with cost function [2][12]. However, it is doubtful for the usage of optimization in our actual neural system. Except the usage of cost function, the simple control paradidm has not yet been reported.

Recently, the novel learning scheme named Tacit learning has been proposed [10][11]. Tacit learning is one of the biomimetic learning architecture where the primitive behaviors composed of the reflexive actions are tuned to the behaviors adapted to the environment taking the environmental information into the controller through body/environment interactions. The experimental result demonstrated that the walking gait composed of the primitive motions of the swing leg was well adapted to the environment in terms of the walking efficiency [11].

In this paper, we propose an optimal control paradigm in motor learning of reaching task which has computational adaptability and optimality without using cost-function based optimization. We demonstrate that simple tacit learning can realize both environmental adaptation and optimal control synchronously in vertical reaching task. The some simulation results with redundant actuators indicated that the proposed method can systematically produce motor synergies and energy efficiency in vertical reaching task which involves complex interaction torques.

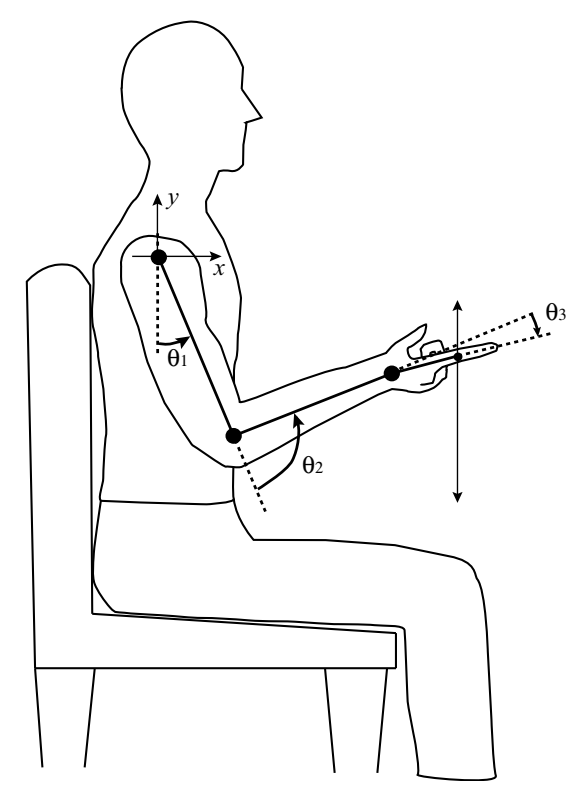

Fig. 1. Schematic representation of vertical reaching task. 


\section{TACIT LEARNING FOR VERTICAL REACHING}

\section{A. Vertical reaching and its dynamics simulation}

In this work, we verify the performance of tacit learning in vertical reaching as shown in Fig. 1. This configuration was used in [1]. In a patient with cerebellar damage, it was difficult to control the end point of the arm in a synergetic way among multiple joints due to the gravity and interaction torques. It implies that cerebellar damage affects prediction of interaction torques which can be normally made based on the internal model established from motor learning. In order to compensate interaction torques, motor learning is essential. Thus, we evaluate the proposed computational learning paradigm in this vertical reaching task.

For the joint dynamics simulation, we have used MatODE [3] which is a Matlab interface to the Open Dynamics Engine [8]. In a sagittal 2D plane, 3 Degrees-of-Freedom (DOF) composed of shoulder, elbow, wrist joint was assumed. The upper arm, forearm and hand segments were connected through each joint in the dynamics simulation environment. Thus, we have an access only to the control of each joint torque and no access to the manipulator dynamics model in the learning process. It should be noted that this configuration is in so-called Bernstein's DOF problem where we have actuation redundancy in the task space.

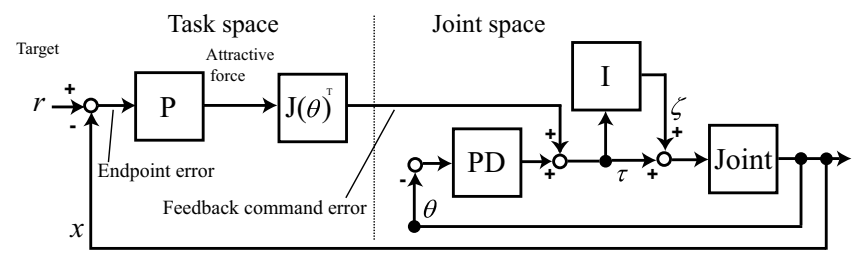

Fig. 2. Motor learning for reaching motion by tacit learning. Task space command by artificial field. Feedback command error and local PD control input are integrated.

\section{B. Controllers in Tacit Learning}

In Tacit Learning, the environmental signal accumulations play the main role to create the appropriate behaviors. In biological controllers, signal accumulations can be considered as the typical learning method to create the behaviors adapted to the environment such as long term depression and augmentation in cerebellum.

In the previous tacit learning for biped walking, joints were divided into kinematically specified ones and unspecified group. Then, unspecified joints were controlled with tacit learning. In this work for reaching task, only the desired position of the endpoint is given, and all joints were controlled with tacit learning as in Fig. 2.

The left side of this figure corresponds to the task space feedback. The linear attractive field is used as the intention of the subject which tries to follow the target. The right side corresponds to the tacit learning in joint space. Torque signal accumulations are performed with local PD controller and feedback command error from the task space. Only kinematics is assumed as known through Jacobian of the arm to map joint space and task space. All dynamical parameters such as segment inertia and mass, and the model itself are completely blind.

The controllers for tacit learning are expressed as follows:

$$
\boldsymbol{\tau}=-\boldsymbol{J}^{T}(\boldsymbol{\theta}) k \Delta \boldsymbol{x}-\boldsymbol{A} \Delta \boldsymbol{\theta}-\boldsymbol{B} \dot{\boldsymbol{\theta}}+\boldsymbol{C} \int \boldsymbol{\tau} d t .
$$

where, $\boldsymbol{\tau}$ implies the torques of joints, $\boldsymbol{\theta}$ implies the angles of joints. $\boldsymbol{J}^{T}(\boldsymbol{\theta})$ is the transpose of the Jacobian of the arm, $\mathrm{k}$ is the intensity of the attractive field, $\Delta \boldsymbol{x}$ is the endpoint error. $\boldsymbol{A}$ and $\boldsymbol{B}$ consist of the proportional and derivative gains of PD controllers. $\boldsymbol{C}$ consists of the integral gains of the output torque integral. The term $\boldsymbol{A} \Delta \boldsymbol{\theta}$ is optional, it can be set if you specify the neutral position of the joint. Note that all joints are controlled independently.

\section{EMERGENCE OF MOTOR SYNERGY}

\section{A. Simulation Study}

In order to evaluate the performance of the proposed tacit learning, we have compared the control results of vertical reaching between (a) PD controller and (b) tacit learning with PD controller. The difference is the existence of the last term in (1). The target point in the coordinate system of Fig.1 was given as below:

$$
\boldsymbol{r}(t)=\left[\begin{array}{ll}
0.3 & -0.3-0.2 \sin (2 \pi f t)
\end{array}\right]^{T} .
$$

It is a task to move his finger tip vertically between two points in the frequency $\mathrm{f}=0.5 \mathrm{~Hz}$. Initial joint angles are $\theta_{1}=$ $0^{\circ}, \theta_{2}=90^{\circ}, \theta_{3}=0^{\circ}$. As for the segment length, the inertia around $\mathrm{z}$ axis and the mass of the upper arm, the forearm and the hand, they are set respectively as follows:

$$
\begin{aligned}
& l_{1}=0.31[\mathrm{~m}] l_{2}=0.27[\mathrm{~m}] l_{3}=0.1[\mathrm{~m}], \\
& I_{1}=0.0141\left[\mathrm{kgm}^{2}\right] I_{2}=0.0120\left[\mathrm{kgm}^{2}\right] I_{3}=0.001\left[\mathrm{kgm}^{2}\right], \\
& m_{1}=1.93[\mathrm{~kg}] m_{2}=1.32[\mathrm{~kg}] m_{3}=0.35[\mathrm{~kg}]
\end{aligned}
$$

In this study, the value of 30 percent of the above mass was used to have the convergence of the learning within 60s.
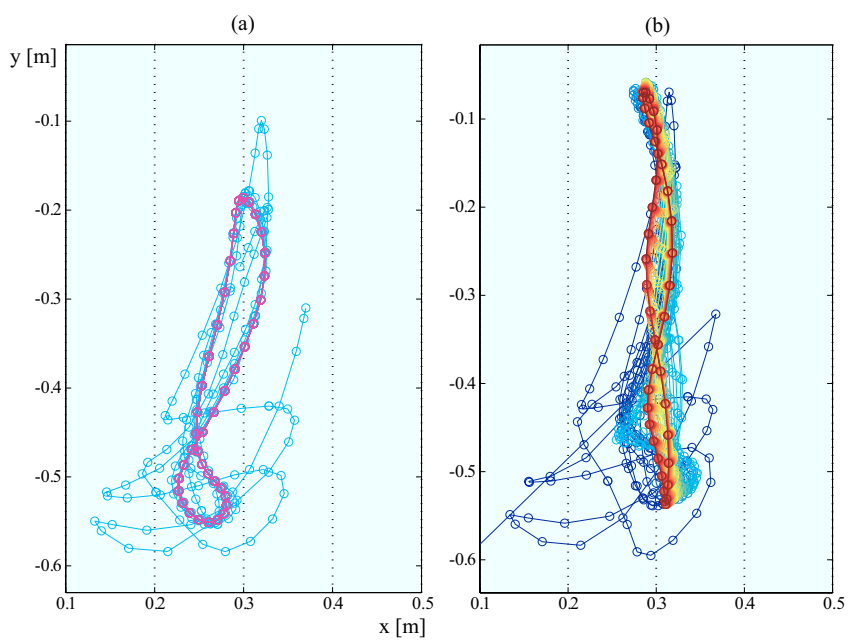

Fig. 3. End point transition. (a) only with PD control (b) with tacit learning in addition to the PD control. 

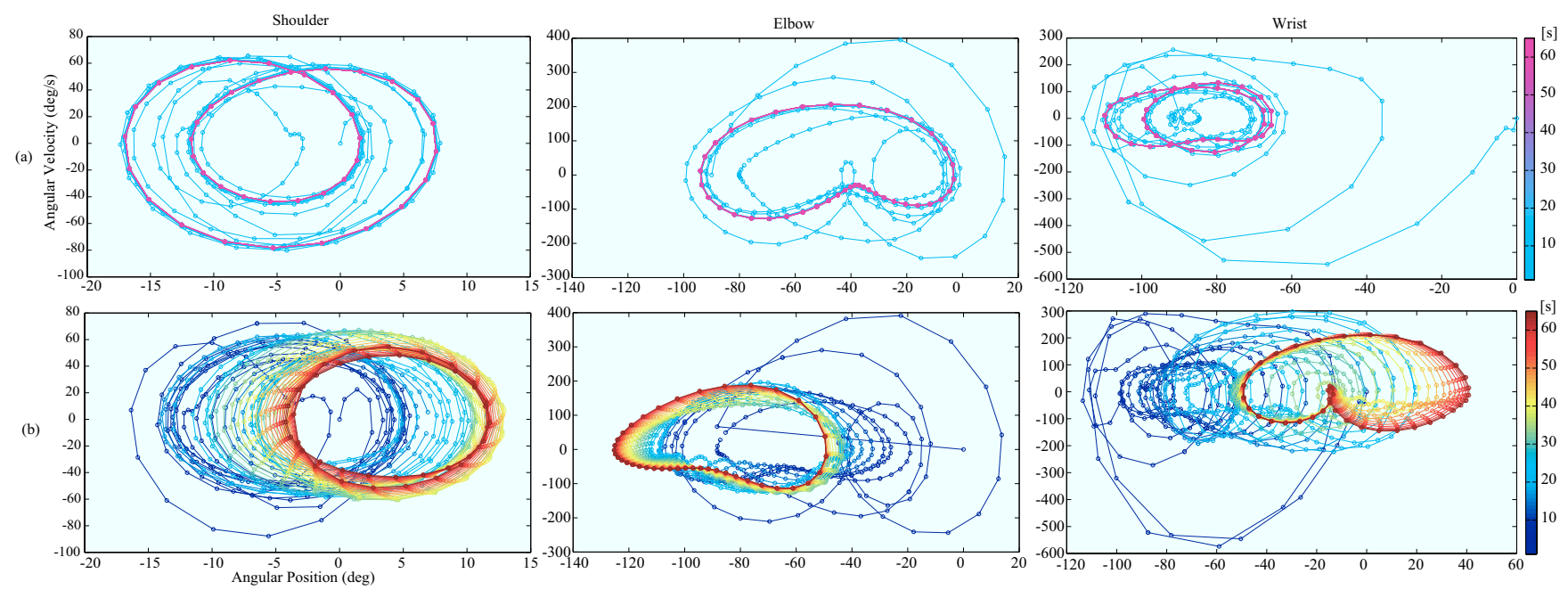

Fig. 4. Phase portrait of shoulder, elbow and wrist joint in joint angle-angular velocity.(a) only with PD control (b) with tacit learning in addition to the PD control. We can find tacit learning leads to synergetic motion with effective one-loop orbit in each joint.

\section{B. Control Results}

Fig. 3 represents a control result of vertical reaching. The plot in Fig. 3(a) is the end point in the case only with PD control, the plot in Fig. 3(b) is with tacit learning in addition to the PD control. The time sequential transition is illustrated using color map which changes depending on the time progress. The color map correspondence to time can be seen in the color bar in the right side of Fig. 4. A cool color map is used for (a) PD control, a jet color map is used for (b) tacit learning. This colormap configuration is used also in other figures in this paper.

Fig. 3(a) shows that PD control is largely affected by the gravity and the interaction torques. On the contrary, we can find that the trajectory is being corrected in time in the case of tacit learning minimizing the effect of the gravity and interaction torque. Fig. 4 shows a phase portrait of shoulder, elbow and wrist joint in joint angle-angular velocity. We can find tacit learning leads to synergetic motion with effective one-loop orbit in each joint. On the contrary, the phase portrait in PD control is not one-loop orbit. It implies that one joint motion conflicts with other joints motion. In tacit learning case, it is interesting to see such synergetic solution
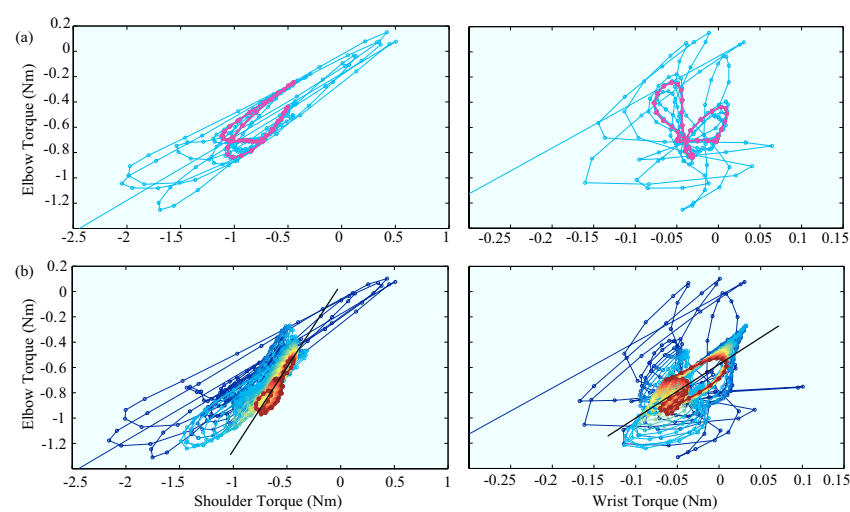

Fig. 5. Torque phase portrait of shoulder-elbow and wrist-elbow joint.(a) only with PD control (b) with tacit learning in addition to the PD control. We can find tacit learning leads to synergetic torque production compared to the PD control of individual joint. is being found with dynamics model-free and cost-functionfree approach. Fig. 5 indicates a torque phase portrait of shoulder-elbow and wrist-elbow joint. We can find the torque production in tacit learning is converged into more aligned synergetic solutions compared to the case in PD control of individual joint. It implies that the tacit learning allowed to learn how to manage the interaction torques and find synergetic combination between neighbouring joints to bring an efficiency of multijoint coordination.

Shoulder joint torque solution is depicted in Fig. 6. The plot in cool color map represents the controlled torque only with PD controller. The plot in jet color map is the controlled torque with tacit learing. We can find that the torque amplitute in the tacit learning is much less than the one in PD controller. It implies that tacit learning has also the function to minimize the torque production. Energy consumption in one cycle of reaching was measured and compared between PD control and tacit learning. The vertical reaching task in this study was the motion between two vertically located points in the frequency $0.5 \mathrm{~Hz}$. Therefore, the energy consumption during every 2 second was calculated by summing up each joint energy consumption $2 \pi \tau \dot{\theta}$. The energy consumption transition along with the time progress is summarized in Table I. We can notice that the energy

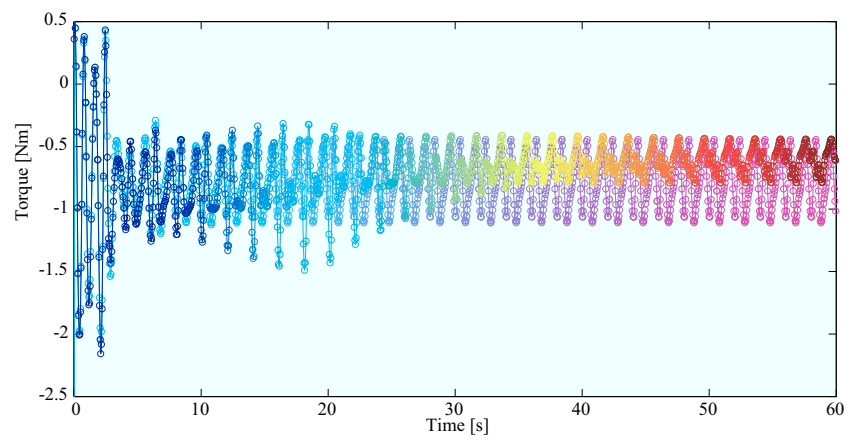

Fig. 6. Shoulder joint torque solution only with PD control (cool color map) with tacit learning in addition to PD control (jet color map). We can find tacit learning leads to minimizing torque production. 
consumption is lower in tacit learning and is being minimized in the course of motor learning while the energy transition is stayed constant in the case of PD control since there is no adaptive functionality. We should note that the vertical displacement of the end point is less in PD control as in Fig. 3. Thus, the actual difference of energy efficiency is much larger than the value in this table. The vertical displacement is longer with 25 percent in tacit learning. Then the energy consumption also can be higher with 25 percent, however the actual reaching task could be performed with even less energy than PD control thanks to the synergetic motor solutions in tacit learning.

TABLE I

ENERGY CONSUMPTION IN ONE CYCLE OF REACHING

\begin{tabular}{|c||c|c|c|c|c|c|c|}
\hline Time (s) & 2 & 10 & 20 & 30 & 40 & 50 & 60 \\
\hline PD & $30.1 \mathrm{~J}$ & $20.3 \mathrm{~J}$ & $20.0 \mathrm{~J}$ & $20.0 \mathrm{~J}$ & $20.0 \mathrm{~J}$ & $20.0 \mathrm{~J}$ & $20.0 \mathrm{~J}$ \\
\hline Tacit & $31.3 \mathrm{~J}$ & $20.0 \mathrm{~J}$ & $18.8 \mathrm{~J}$ & $19.5 \mathrm{~J}$ & $18.4 \mathrm{~J}$ & $18.3 \mathrm{~J}$ & $18.2 \mathrm{~J}$ \\
\hline
\end{tabular}

\section{CONCLUSION AND DISCUSSION}

In this paper, we have proposed a novel computational control paradigm in motor learning of vertical reaching task which involves the management of interaction torques and gravitational effect. From the control result in vertical reaching, we claim that the proposed method is valid for acquiring motor synergy in the system with actuation redundancy. We should highlight that the tacit learning in vertical reaching task bring computational adaptability and optimality with model-free and cost-function-free approach differently from previous studies. Energy efficient solutions could be obtained by the emergence of motor synergy in the redundant actuation space.

In addition, the simulation result obtained in this paper showed good correspondence to the experimental result in [1]. In their experiment, they found that the inability to produce accommodative joint torque for the dynamic interaction torques appears to be an important cause of kinematic deficits shown by subjects with cerebellar abnormality. Thus, the reaching by them included incoordination of the shoulder and the elbow joints, a curved endpoint trajectory according to [1]. Those characteristics of reaching in subjects with cerebellar abnormality is equivalent to the result (a) only with PD control in this study. On contrary, the experimental reaching in able-bodied subject showed the correspondence to the result (b) with tacit learning. In [1], they suggest that a major role of cerebellum is in generating joint torques at a joint that will predict the interaction torques being generated by other moving joints and compensate for them. It implies that the proposed computational learning paradigm represents well the learning principles actually taken place in cerebellum.

Uncovering the unknown mechanism in motor learning has significant meaning in computational rehabilitation. Recently, the some robotics devices or neuroprosthetic devices such as functional electrical stimulation are to be used for rehabilitation of the patients with motor disabilities. Rehabilitation is a sort of motor learning in the sense where the patients need to find new neural path way to achieve certain task. By means of taking advantage of the hidden principles in our motor learning, the effective rehabilitation protocol would be designed along with the computer-aided approach.

\section{REFERENCES}

[1] A J Bastian, T A Martin, J G Keating, and W T Thach. Cerebellar ataxia: abnormal control of interaction torques across multiple joints. Journal of Neurophysiology, 76(1):492-509, 1996.

[2] Daniel a Braun, Ad Aertsen, Daniel M Wolpert, and Carsten Mehring. Learning optimal adaptation strategies in unpredictable motor tasks. The Journal of neuroscience : the official journal of the Society for Neuroscience, 29(20):6472-8, May 2009.

[3] Wouter Caarls. MatODE, http://wouter.caarls.org/matode.html, 2010.

[4] P L Gribble and D J Ostry. Compensation for interaction torques during single- and multijoint limb movement. Journal of neurophysiology, 82(5):2310-26, November 1999.

[5] M Ito. Neural design of the cerebellar motor control system. Brain Research, 40(1):81-84, 1972.

[6] M Kawato and H Gomi. A computational model of four regions of the cerebellum based on feedback-error learning. Biological cybernetics, 68(2):95-103, January 1992.

[7] M Kawato and H Gomi. The cerebellum and VOR/OKR learning models. Trends in Neurosciences, 15(11):445-453, 1992.

[8] Russell Smith. Open Dynamics Engine, http://www.ode.org/, 2000.

[9] Reza Shadmehr and Steven P Wise. The computational neurobiology of reaching and pointing: a foundation for motor learning. MIT Press, 2005.

[10] S Shimoda and H Kimura. Biomimetic Approach to Tacit Learning Based on Compound Control. Systems, Man, and Cybernetics, Part B: Cybernetics, IEEE Transactions on, 40(1):77-90, 2010.

[11] S Shimoda, Y Yoshihara, and H Kimura. Emergence of bipedal walking through body/environment interactions. In Intelligent Robots and Systems (IROS), 2010 IEEE/RSJ International Conference on, pages $1760-1765,2010$.

[12] Emanuel Todorov. Optimality principles in sensorimotor control. Nature neuroscience, 7(9):907-15, September 2004.

[13] Y. Uno, M. Kawato, and R. Suzuki. Formation and control of optimal trajectory in human multijoint arm movement. Minimum torquechange model. Biological cybernetics, 61:89-101, 1989.

[14] D M Wolpert, R C Miall, and M Kawato. Internal models in the cerebellum. Trends in cognitive sciences, 2(9):338-47, September 1998. 